\title{
Meta-analysis of randomized controlled trials comparing $17 \alpha$-hydroxyprogesterone caproate and vaginal progesterone for the prevention of recurrent spontaneous preterm delivery
}

\author{
Elizabeth Oler $^{1}$ | Ahizechukwu C. Eke ${ }^{2, *}$ | Ashley Hesson ${ }^{3}$
}

\author{
${ }^{1}$ Department of Obstetrics and \\ Gynecology, Johns Hopkins University School \\ of Medicine, Baltimore, MD, USA \\ ${ }^{2}$ Division of Maternal Fetal \\ Medicine, Department of Obstetrics and \\ Gynecology, Johns Hopkins University School \\ of Medicine, Baltimore, MD, USA \\ ${ }^{3}$ Department of Obstetrics and \\ Gynecology, University of Michigan School of \\ Medicine, Ann Arbor, MI, USA

\section{${ }^{*}$ Correspondence} \\ Ahizechukwu C. Eke, Division of Maternal \\ Fetal Medicine, Department of Obstetrics and \\ Gynecology, Johns Hopkins University School \\ of Medicine, Baltimore, MD, USA. \\ Email: aeke2@jhu.edu
}

\begin{abstract}
Background: Vaginal progesterone and $17 \alpha$-hydroxyprogesterone (17 $\alpha-\mathrm{OHP})$ are both used to prevent preterm delivery in women who have experienced spontaneous preterm delivery (SPTD) previously. Randomized trial data of the comparative effectiveness of these interventions have been mixed.

Objectives: To compare the efficacy of intramuscular $17 \alpha-\mathrm{OHP}$ and vaginal progesterone in the prevention of recurrent SPTD.

Search strategy: Cochrane Central Register of Controlled Trials, African Journals Online, Embase, Google Scholar, ISI Web of Science, LILACS, CINAHL, PubMed, and registers of ongoing trials were searched using keywords related to $17 \alpha-\mathrm{OHP}$, vaginal progesterone, and preterm delivery.

Selection criteria: Randomized controlled trials published between January 1, 1966, and November 30, 2016, comparing $17 \alpha-\mathrm{OHP}$ and vaginal progesterone for the prevention of recurrent SPTD during singleton pregnancies were included.

Data collection and analysis: Study data were extracted and meta-analyses were performed when outcomes were comparable.

Main results: The meta-analyses included data from three randomized trials. Lower rates of SPTD before 34 weeks (relative risk 0.71 , 95\% confidence interval 0.53-0.95) and before 32 weeks (relative risk 0.62 , 95\% confidence interval $0.40-0.94$ ) of pregnancy were observed among patients treated with vaginal progesterone.

Conclusions: Vaginal progesterone and $17 \alpha-\mathrm{OHP}$ were comparable for the prevention of recurrent SPTD in singleton pregnancies; vaginal progesterone could be superior.

KEYWORDS

17 alpha progesterone; Preterm labor; Recurrent preterm delivery; Vaginal progesterone
\end{abstract}

\section{1 | INTRODUCTION}

Preterm delivery has become a major public health problem owing to its contribution to maternal adverse events as well as neonatal morbidity and mortality; despite its decreasing incidence in the USA, it is a leading cause of long-term disability. ${ }^{1}$ In the Centers for Disease Control and Prevention National Center for Health Statistics delivery data for $2013,{ }^{2}$ the preterm delivery rate had decreased to $11.39 \%$ of all live deliveries. The use of progesterone therapy-vaginal progesterone and intramuscular $17 \alpha$-hydroxyprogesterone acetate-has been demonstrated to be a major contributor to the decreasing incidence of preterm delivery. ${ }^{3}$

Based on randomized clinical trial data, ${ }^{4}$ intramuscular $17 \alpha-$ hydroxyprogesterone (17 $\alpha$-OHP) has been approved by the US Food 
and Drug Administration for the prevention of recurrent preterm delivery in patients who have experienced preterm delivery previously. Data from 459 women with singleton pregnancies and a history of spontaneous preterm delivery (SPTD) at $20-36^{+6}$ weeks of a singleton pregnancy were included and the efficacy of $17 \alpha-\mathrm{OHP}$ was compared with placebo. Weekly 250-mg doses of intramuscular $17 \alpha-\mathrm{OHP}$, started at $16-20^{+6}$ weeks of pregnancy, were associated with a reduced incidence of SPTD prior to 35 weeks, 37 weeks, and 32 weeks of pregnancy. ${ }^{4}$ In comparison with placebo, $17 \alpha$-OHP was also associated with significant decreases in neonatal oxygen supplementation and intraventricular hemorrhage. ${ }^{4}$ Based primarily on these data, ${ }^{4} 17 \alpha-\mathrm{OHP}$ has been recommended for all women with a history of SPTD at $20-36^{+6}$ weeks of pregnancy. ${ }^{5}$

By contrast, vaginal progesterone has only been recommended for use in the prevention of SPTD in patients with no history of SPDT and with a short cervix identified incidentally during the second trimester of pregnancy. However, the multicenter randomized controlled trial conducted by Fonseca et al. ${ }^{6}$ demonstrated that vaginal progesterone was effective in patients without shortened cervical length. This study reported that nightly 200-mg doses of vaginal progesterone, started at 24 weeks of pregnancy and continuing until 34 weeks, was associated with a significant decrease in SPTD before 34 weeks of pregnancy irrespective of cervical length. ${ }^{6}$ Notably, no increased benefit was recorded among patients with a history of at least one SPTD.

Current guidelines for patients with a history of SPTD and no evidence of a shortened cervix vary. The American College of Obstetricians and Gynecologists recommends progesterone administration for the prevention of subsequent SPTDs but does not recommend a specific progesterone formulation. ${ }^{5}$ The US Food and Drug Administration has approved only intramuscular progesterone for the treatment of these patients; this stance is based largely on the study of Meis et al. ${ }^{4}$ from 2003 . There have only been several studies that have made head-to-head comparisons of intramuscular and vaginal progesterone to prevent recurrent preterm delivery in patients with normal or unknown cervical length. ${ }^{7-9}$ Further, to the best of our knowledge no known systematic reviews or meta-analyses have compared the efficacy of intramuscular $17 \alpha-\mathrm{OHP}$ and vaginal progesterone, despite randomized trial data being available. Therefore, the aim of the present study was to compare the efficacy of $17 \alpha-\mathrm{OHP}$ and vaginal progesterone in preventing SPTD in patients who have experienced SPTD previously.

\section{2 | METHODS}

The present study was performed in accordance with the Preferred Reporting Items for Systematic Reviews and Meta-analyses (PRISMA) guidelines. ${ }^{10}$

\section{1. | Literature search}

The African Journals Online (AJOL), Cochrane Central Register of Controlled Trials, Embase, Google Scholar, ISI Web of Science,
LILACS, CINAHL, and PubMed databases, and several clinical trial registries (http://www.nihr.ac.uk; www.clinicaltrials.gov; www.umin. ac.jp/ctr; www.anzctr.org.au; www.controlled-trials.com; and www. centerwatch.com) were searched for randomized controlled trials that compared intramuscular $17 \alpha-\mathrm{OHP}$ and a vaginal formulation of progesterone. Databases were searched from inception until November 30, 2016. The terms "17 alpha hydroxyprogesterone caproate", "vaginal progesterone", "prometrium", "Makena", "crinone", "prochieve", "endometrin", "preterm labor", "recurrent", "spontaneous preterm", "preterm contractions", and "preterm birth" were used to search for articles. Proceedings of international society meetings where preterm delivery was an identified focus were also considered. If multiple versions of publications were available, the most complete, fully supplemented versions were reviewed where available.

\subsection{Study selection}

Randomized clinical trials that included vaginal progesterone and intramuscular $17 \alpha-\mathrm{OHP}$ as interventions for the prevention of recurrent preterm delivery of singleton pregnancies irrespective of cervical length were included. The exclusion criteria were: (1) if progestational agents were used for tocolysis and not specifically to prevent recurrent preterm delivery in asymptomatic patients; (2) if two form of vaginal progesterone were compared for the prevention of preterm delivery; (3) if trials were quasi-randomized; and (4) if English translations were unavailable for foreign-language articles. Published abstracts without accompanying full-text manuscripts were excluded if there was insufficient detail to determine study eligibility. Authors of published manuscripts were contacted if additional information on study methods were needed. Qualifying studies were reviewed independently by two researchers (A.C.E. and E.O.) with subsequent discussion to reach ultimate inclusion decisions if necessary.

\section{3 | Outcome measures}

The primary outcome measure was delivery occurring before 34 weeks of pregnancy. The secondary outcomes were delivery before 37 weeks of pregnancy, delivery before 32 weeks of pregnancy, delivery before 28 weeks of pregnancy, delivery before 24 weeks of pregnancy, low delivery weight $(<2500 \mathrm{~g})$, need for mechanical ventilation, neonatal intensive care unit admission, 5-minute Apgar scores below seven, perinatal death, neonatal morbidity (respiratory distress syndrome, intraventricular or intracerebral bleeding, neonatal sepsis, or necrotizing enterocolitis), maternal adverse events, recurrent preterm labor, and discontinuation of treatment owing to adverse events.

\subsection{Assessment of risk of bias}

Study quality and risk of bias were assessed using Cochrane Collaboration standards. ${ }^{11}$ This approach considers seven factors: (1) concealment of allocation; (2) random sequence generation; (3) successive masking of participants and personnel to experimental and 
control medications; (4) masking of outcome assessment; (5) incomplete outcome data description; (6) selective reporting of data; and (7) other bias. Bias assessments were made by two researchers (A.C.E. and E.O.) and were categorized as "low risk", "high risk", or "unclear risk" of bias; collaborative resolution was used for any disagreements.

\section{5 | Data extraction}

A standardized abstraction form was used to record data from the studies included. The randomization method, method for concealing allocation, individuals masked to treatment (providers, patients, and outcome assessors), participant data (trial location, demographic characteristics, inclusion and exclusion criteria, definition of recurrent SPTD, duration of pregnancy at randomization, cervical dilation, and effacement at trial entry), number of patients randomized, details of interventions (administration route, dose details, and treatment duration), and outcomes were coded by two researchers independently (A.C.E. and E.O.). Again, differences in coding were discussed until consensus was achieved.

\section{6 | Statistical analysis}

Cochrane Collaboration guidelines ${ }^{12}$ were followed when performing intent-to-treat analyses. Meta-analyses were only performed if study populations, interventions, and outcome measures were comparable. Summary relative risks (RRs) were calculated for dichotomous data. Mean differences were calculated for continuous data if a given outcome was measured similarly across trials and standardized mean differences were used if there was variation in the way an outcome was measured between studies.

Ultimately, no subgroup analyses were performed. A subgroup analysis to compare vaginal progesterone and intramuscular $17 \alpha-\mathrm{OHP}$ with different types of vaginal progesterone was planned; however, it could not be performed owing to a lack of trials being identified that included these comparisons. Similarly, additional subgroup analyses of progesterone dose, duration of pregnancy at trial entry, and study setting were abandoned owing to low cell sizes.

The heterogeneity of study data was measured using $l^{2}$, which describes the percentage of total variation across studies that was due to heterogeneity and not chance. ${ }^{13} \mathrm{I}^{2}$ values of at least $50 \%$ indicate substantial heterogeneity, values below $30 \%$ indicate low

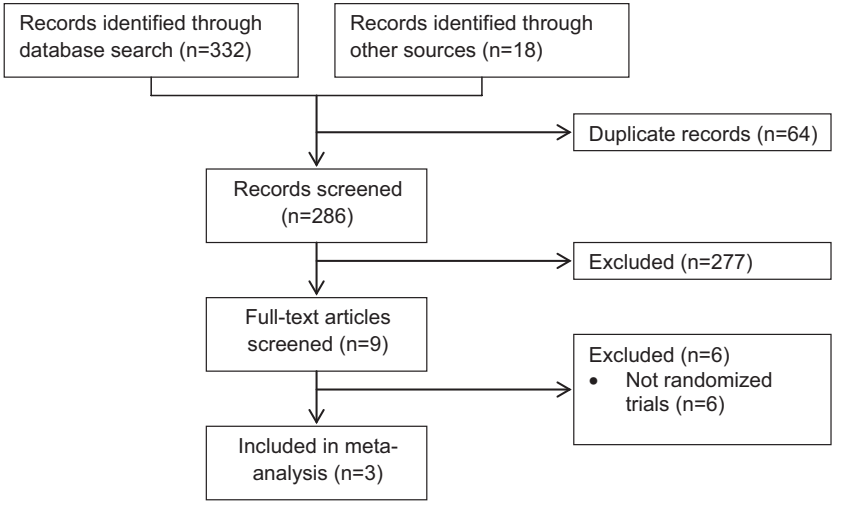

FIGURE 1 Study selection.

heterogeneity, and values of $30-50 \%$ indicate moderate heterogeneity. ${ }^{13} \mathrm{~A}$ fixed-effects model was considered appropriate for pooling studies when $I^{2}$ was above $50 \%$; a random-effects model was used for pooling other variables. The Egger test (with a cut-off value of $<0.1$ ) and visually assessed funnel-plot symmetry were used to assess publication bias. ${ }^{13}$ All analyses were performed with RevMan version 5.3.5 (The Nordic Cochrane Centre, Copenhagen, Denmark) and $P<0.05$ was considered statistically significant.

\section{3 | RESULTS}

There were three studies that met the inclusion criteria for the metaanalysis (Fig. 1) including a total of 680 patients with a history of preterm delivery (Table 1). Of these, 332 (48.8\%) patients were randomized to treatment with $17 \alpha-\mathrm{OHP}$ and $348(51.2 \%)$ to treatment with vaginal progesterone. The mean duration of pregnancy at randomization was 16 weeks. Treatment continued until 36 weeks of pregnancy or delivery.

Delivery before 34 weeks of pregnancy was less frequent among patients treated with vaginal progesterone compared with those treated with $17 \alpha-\mathrm{OHP}(19.3 \%$ vs $26.7 \%$; RR 0.71 , $95 \%$ confidence interval [Cl] 0.53-0.95) (Fig. 2). Similarly, delivery before 32 weeks of pregnancy was also lower among patients treated with vaginal progesterone (12.4\% vs 16.1\%; RR 0.62, 95\% Cl 0.40-0.94) (Fig. 3). No difference was observed between the two treatment groups for delivery

TABLE 1 Demographic data of studies included in the meta-analysis.

\begin{tabular}{|c|c|c|c|}
\hline Variable & Bafghi et al. $^{7}$ & Elimian et al. ${ }^{8}$ & Maher et al. ${ }^{9}$ \\
\hline Study setting & Iran & United States & Saudi Arabia \\
\hline Primary outcome measure & Duration of pregnancy at delivery & $\begin{array}{l}\text { Spontaneous preterm delivery at } \\
<37 \text { weeks of pregnancy }\end{array}$ & $\begin{array}{l}\text { Spontaneous preterm delivery at } \\
<34 \text { weeks of pregnancy }\end{array}$ \\
\hline No. of patients included & 33 & 145 & 502 \\
\hline Vaginal progesterone & 16 & 79 & 253 \\
\hline Intramuscular progesterone & 17 & 66 & 249 \\
\hline Intramuscular $17 \alpha-\mathrm{OHP}$ dose & 250 mg weekly & 250 mg weekly & 250 mg weekly \\
\hline $\begin{array}{l}\text { Vaginal progesterone dose and } \\
\text { administration }\end{array}$ & 200 mg daily, suppository & 100 mg daily, suppository & 90 mg daily, vaginal gel \\
\hline
\end{tabular}




\begin{tabular}{|c|c|c|c|c|c|}
\hline \multirow[b]{2}{*}{ Study or Subgroup } & \multicolumn{2}{|c|}{ Vaginal progesterone } & \multicolumn{2}{|c|}{ 17-ОНРС } & \multirow[b]{2}{*}{ Weight } \\
\hline & Events & Total & Events & Total & \\
\hline Bafghi 2015 & 5 & 16 & 6 & 17 & $9.2 \%$ \\
\hline Elimian 2016 & 14 & 79 & 13 & 66 & $18.8 \%$ \\
\hline Maher 2012 & 42 & 253 & 64 & 249 & $72.0 \%$ \\
\hline Total $(95 \% \mathrm{Cl})$ & & 348 & & 332 & $100.0 \%$ \\
\hline Total events & 61 & & 83 & & \\
\hline
\end{tabular}

Risk Ratio

Random, 95\% Cl

$0.89[0.34,2.34]$

$0.90[0.46,1.78]$

$0.65[0.46,0.91]$

$0.71[0.53,0.95]$

Test for overall effect: $Z=2.30(P=0.02)$

FIGURE 2 Spontaneous preterm delivery at <34 weeks of pregnancy.

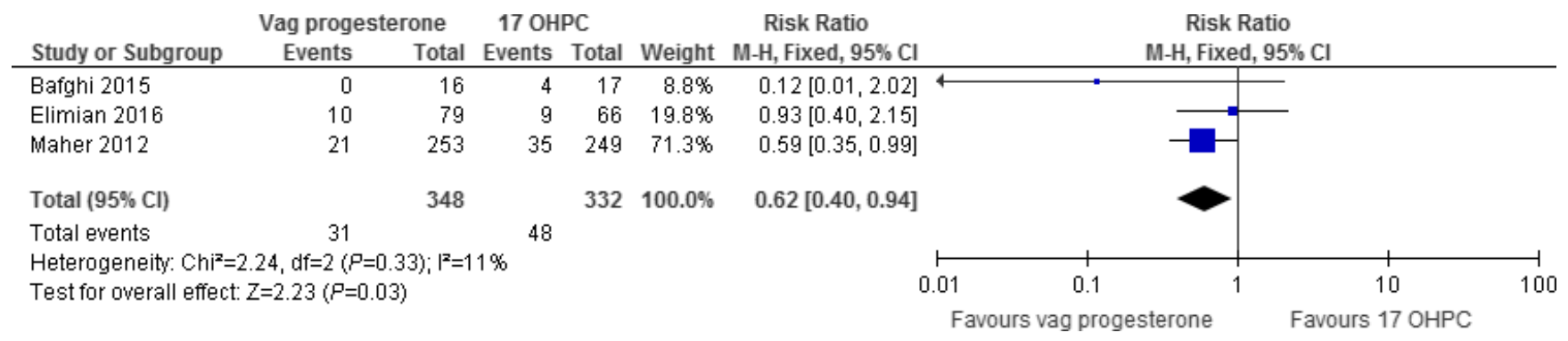

FIGURE 3 Spontaneous preterm delivery at <32 weeks of pregnancy.

before 37 weeks or 28 weeks of pregnancy; however, non-significant trends favoring vaginal progesterone were observed for both outcomes (Figs 4 and 5).

There were no differences between the rate of respiratory distress syndrome in the two groups (RR 1.02, 95\% Cl 0.65-1.60) (Fig. 6) or neonatal admission to the intensive care unit (RR 1.17, 95\% $\mathrm{Cl}$ 0.49-2.79). A lower rate of adverse treatment reactions was observed among patients treated with $17 \alpha-\mathrm{OHP}(12.3 \%$ vs $17.4 \%$; RR $0.53,95 \% \mathrm{Cl} 0.31-0.91$ ); however, this finding was based on data from a single study. Owing to insufficient data, it was not possible to analyze the delivery rate before 24 weeks of pregnancy, recurrent preterm labor, low delivery weight, need for mechanical ventilation, 5-minute Apgar score below seven, perinatal death, or neonatal morbidity (including intraventricular or intracerebral bleeding, neonatal sepsis, and necrotizing enterocolitis).

\section{DISCUSSION}

In the present meta-analysis, patients who were treated with vaginal progesterone demonstrated significantly lower rates of SPTD before 34 weeks of pregnancy and before 32 weeks of pregnancy

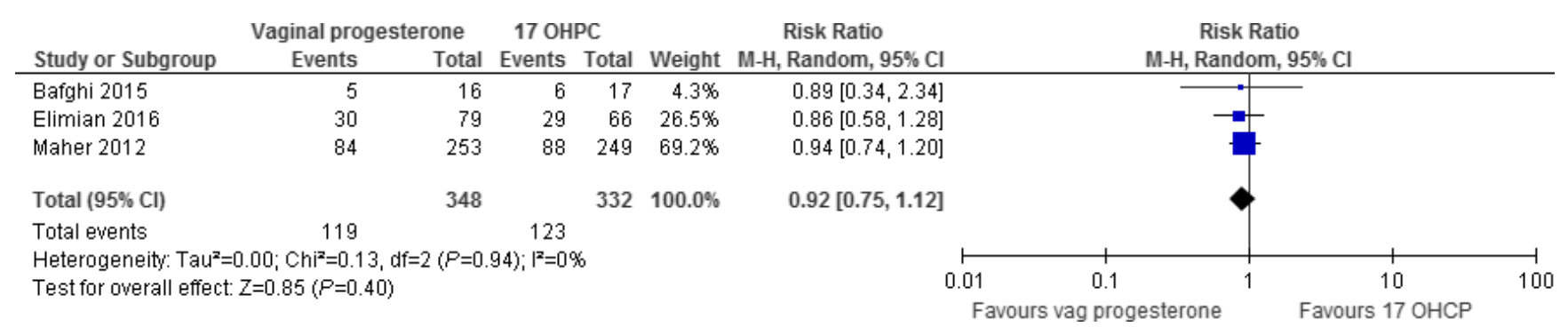

FIGURE 4 Spontaneous preterm delivery at < 37 weeks of pregnancy.

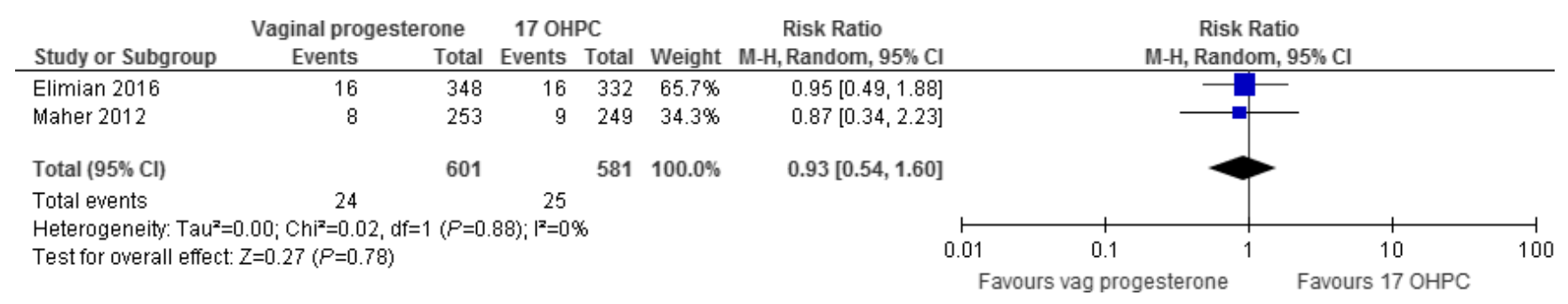

FIGURE 5 Spontaneous preterm delivery at $<28$ weeks of pregnancy. 


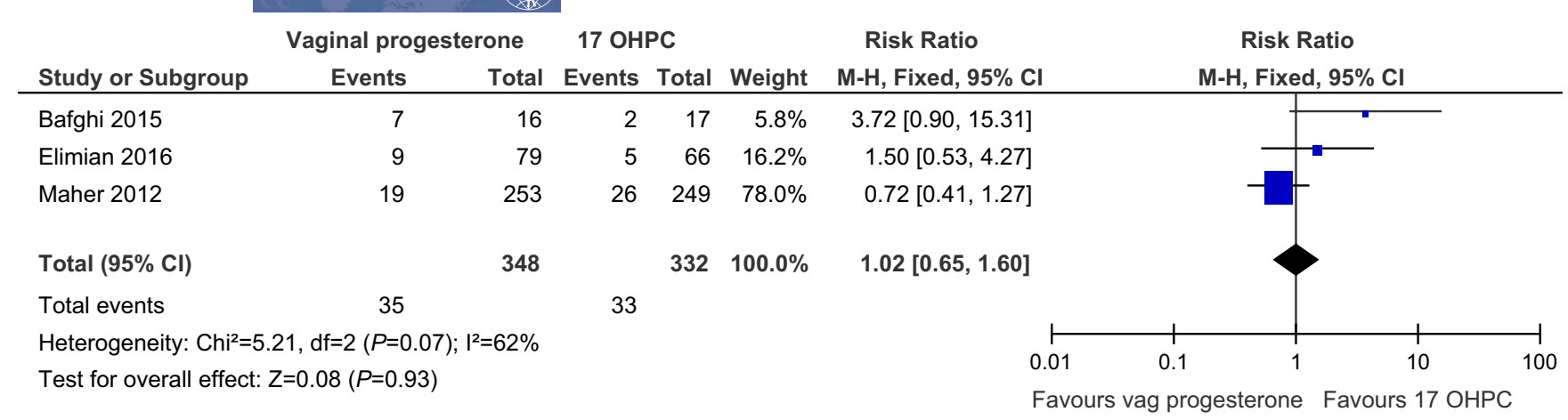

FIGURE 6 Respiratory distress syndrome.

compared with patients treated with $17 \alpha-\mathrm{OHP}$. To the best of our knowledge, the present meta-analysis was the first to compare outcomes from randomized controlled trials assessing the efficacy of prophylactic vaginal progesterone and intramuscular progesterone in patients with singleton pregnancies and a history of SPTD, regardless of cervical length. Contrary to a subset of currently available guidelines for progesterone administration, the present study found that vaginal and intramuscular formulations were at least comparable in their efficacy preventing recurrent SPTD and preventing some associated forms of neonatal morbidity. In fact, vaginal progesterone could even be superior to intramuscular $17 \alpha-\mathrm{OHP}$ in decreasing the risk of delivery prior to 34 weeks of pregnancy. Similarly, the vaginal formulation could have a more favorable risk profile, based on the outcomes measured.

There were several limitations in the present study. First, each trial used different formulations of vaginal progesterone. Unfortunately, the safest and most effective dose of vaginal progesterone for SPTD prophylaxis has not been established. This variation limits the validity of the results. Second, the individual studies were limited by the low participant numbers and secondary outcome heterogeneity. However, a strength of all three studies included was the stringent randomization, without significant demographic differences between the treatment groups.

As there were only three studies in the meta-analysis, many secondary outcomes could not be assessed. Notably, these included perinatal death and the need for mechanical ventilation, complicating the assessment of the neonatal mortality and severe morbidity of vaginal and intramuscular progesterone treatment.

Ultimately, the present meta-analysis, despite some unavoidable flaws, has shed light on a possible alternative to intramuscular progesterone, the current gold-standard treatment for patients with a history of preterm delivery. Further randomized controlled trials are certainly warranted to explore this question further.

\section{AUTHOR CONTRIBUTIONS}

EO contributed to performing the literature review and writing the manuscript. ACE contributed to the conception of the study, the literature review, data analysis, and writing the manuscript. $\mathrm{AH}$ contributed to the literature review, data analysis, and revising the manuscript.

\section{CONFLICTS OF INTEREST}

The authors have no conflicts of interest.

\section{REFERENCES}

1. Goldenberg RL, Culhane JF, lams JD, Romero R. Epidemiology and causes of preterm birth. Lancet. 2008;371:75-84.

2. Martin JA, Hamilton BE, Osterman MJK, Curtin SC, Mathews TJ. Centers for Disease Control and Prevention National Center for Health Statistics National Vital Statistics System births: Final data for 2013. Natl Vital Stat Rep. 2015;64:1-68.

3. Callaghan WM, MacDorman MF, Rasmussen SA, Qin C, Lackritz EM. The contribution of preterm birth to infant mortality rates in the United States. Pediatrics. 2006;118:1566-1573.

4. Meis PJ, Klebanoff M, Thom E, et al. Prevention of recurrent preterm delivery by 17 alpha-hydroxyprogesterone caproate. N Engl J Med. 2003;348:2379-2385.

5. American College of Obstetricians and Gynecologists. Use of progesterone to reduce preterm birth: ACOG committee opinion no. 419. Obstet Gynecol. 2008;112:963-965.

6. Fonseca EB, Celik E, Parra M, Singh M, Nicolaides KH. Progesterone and the risk of preterm birth among women with a short cervix. N Engl J Med. 2007;357:462-469.

7. Bafghi AS, Bahrami E, Sekhavat L. Comparative Study of Vaginal versus Intramuscular Progesterone in the Prevention of Preterm Delivery: A Randomized Clinical Trial. Electron Physician. 2015;7: 1301-1309.

8. Elimian A, Smith K, Williams M, Knudtson E, Goodman JR, Escobedo $\mathrm{MB}$. A randomized controlled trial of intramuscular versus vaginal progesterone for the prevention of recurrent preterm birth. Int J Gynecol Obstet. 2016;134:169-172.

9. Maher MA, Abdelaziz A, Ellaithy M, Bazeed MF. Prevention of preterm birth: A randomized trial of vaginal compared with intramuscular progesterone. Acta Obstet Gynecol Scand. 2013;92:215-222.

10. Liberati A, Altman DG, Tetzlaff J, et al. The PRISMA statement for reporting systematic reviews and meta-analyses of studies that evaluate health care interventions: Explanation and elaboration. Ann Intern Med. 2009;151:W65-W94.

11. Higgins JPT, Altman DG, Sterne JAC. Chapter 8: Assessing risk of bias in included studies. In: Higgins JPT, Green S, eds. Cochrane Handbook for Systematic Reviews of Interventions Version 5.1.0 (updated March 2011). Chichester: The Cochrane Collaboration; 2011.

12. Deeks JJ, Higgins JPT, Altman DG. Chapter 9: Analyzing data and undertaking meta-analyses. In: Higgins JPT, Green S, eds. Cochrane Handbook for Systematic Reviews of Interventions Version 5.1.0 (updated March 2011). Chichester: The Cochrane Collaboration; 2011.

13. Higgins JP, Thompson SG, Deeks JJ, Altman DG. Measuring inconsistency in meta-analyses. BMJ. 2003;327:557-560. 